\section{Variability of Left Ventricular Outflow Tract Gradient in Hypertrophic Cardiomyopathy}

Geske JB et al.: Clin Cardiol 2009;32(7):397-402

\section{To the editor:}

The degree of left ventricular outflow tract (LVOT) obstruction is dynamic and greatly dependent upon ventricular load and contractility. In a recent publication analyzing LVOT gradient variability, Geske et $\mathrm{al}^{1}$ studied patients who underwent comprehensive 2-dimensional Doppler transthoracic echocardiography and cardiac catheterization with a transseptal measurement of left-sided pressures. All studies were performed within 48 hours of one another. The marked LVOT gradient variability was confirmed because the correlation of LVOT gradients from both methods performed at different times had a wide scatter with $95 \%$ confidence limits of agreement $( \pm 84 \mathrm{~mm} \mathrm{Hg}$ ). Investigators performed an additional substudy in 15 patients with a simultaneous measurement of the LVOT gradient, which revealed a very strong correlation $(r=0.98, P<0.0001)$ with $95 \%$ confidence limits of agreement $( \pm 12 \mathrm{~mm} \mathrm{Hg})$. This significant agreement between simultaneous noninvasive and invasive measurements may have resulted from the perfect methodology of left ventricular catheterization. A transseptal approach allows for not only avoiding catheter entrapment (as authors stressed), but also eliminated other mechanisms minimizing the LVOT gradient. Namely, the insertion of the catheter from the aorta into the left ventricle may significantly reduce the LVOT gradient due to a decrease of SAM intensity. The mitral leaflets leaned on the catheter (Figure 1). We observed this phenomenon in 2 patients who were catheterized before alcohol ablation. $^{2}$

The LVOT pressure gradients measured by the echo Doppler method decreased significantly from 142 to 34 and from 97 to $22 \mathrm{~mm} \mathrm{Hg}$ during catheter insertion into the LVOT. The reversibility of this phenomenon was demonstrated because after pulling the catheter out from the left ventricle, the LVOT gradients increased. The LVOT pressure gradient measured in patients with intensive SAM invasively may be artificially underestimated when a transaortic approach is used.

The LVOT variability may be marked from moment to moment. It has been demonstrated that obstruction may also increase due to load reduction after a change of position, from supine to upright. ${ }^{3-5}$ Recently, Vaglio et $\mathrm{al}^{6}$ documented that latent obstruction in hypertrophic cardiomyopathy ( $\mathrm{HCM}$ ) is of adverse prognostic significance, and that the evaluation of HCM patients with resting LVOT
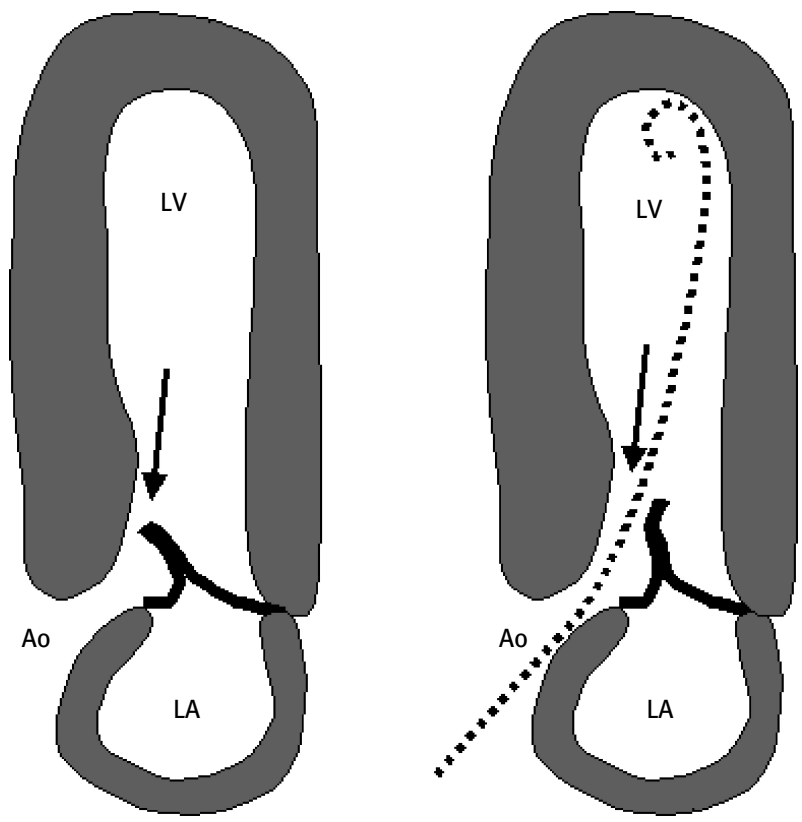

Figure 1. The scheme of the mechanism leading to a decrease of LVOT pressure gradient after insertion of the catheter into the left ventricle in patients with SAM.

gradients $<30 \mathrm{~mm} \mathrm{Hg}$ must include provocative maneuvers to identify this substantial subset of patients, preferably by physiologic exercise. They have concluded that HCM patients with exertional symptoms in the absence of a resting obstruction should undergo additional provocative studies to uncover a possible latent obstruction, and that it is important to recognize that obstruction is augmented in the upright and active positions and minimized in the recumbent position used to obtain echocardiographic images. Measuring LVOT gradients by echocardiography in decubitus, which is the routine practice for assessing patients with $\mathrm{HCM}$, does not reflect the pathophysiology of this pathology during daily activities, which trigger the symptoms that patients report to their cardiologists. Recently, it has been proposed that exercise is the only provocative maneuver that is truly physiologically based and most relevant to conditions under which HCM patients incur symptoms.

Paweł Petkow Dimitrow MD; Wojciech Plazak MD; Piotr Pieniążek MD; Piotr Podolec MD Institute of Cardiology, CMUJ, Cracow, Poland

\section{References}

1. Geske JB, Sorajja P, Ommen SR, Nishimura RA. Left ventricular outflow tract gradient variability in hypertrophic cardiomyopathy. Clin Cardiol. 2009;32:397-402. 
2. Płazak W, Pieniążek P, Podolec P, et al. Left ventricle outflow tract pressure gradient in patients with hypertrophic cardiomyopathy and mitral SAM: echocardiography is superior to invasive measurements. Eur J Echocardiogr. 2004;(Suppl I):58.

3. Cotrim C, Loureiro MJ, Simoes O, et al. Evaluation of hypertrophic obstructive cardiomyopathy by exercise stress echocardiography. New methodology. Rev Port Cardiol. 2005;24:1319-1327.

4. Shah JS, Tome Esteban MT, Thaman R, et al. Prevalence of exercise induced left ventricular outflow tract obstruction in symptomatic patients with non-obstructive hypertrophic cardiomyopathy. Heart. 2008;94:1288-1294.

5. Dimitrow PP, Bober M, Micha lowska J, Sorysz D. Left ventricular outflow tract gradient provoked by upright position or exercise in treated patients with hypertrophic cardiomyopathy without obstruction at rest. Echocardiography. 2009;26:513-520.

6. Vaglio JC, Ommen SR, Nishimura RA, et al. Clinical characteristics and outcomes of patients with hypertrophic cardiomyopathy with latent obstruction. Am Heart J. 2008;156:342-347. 